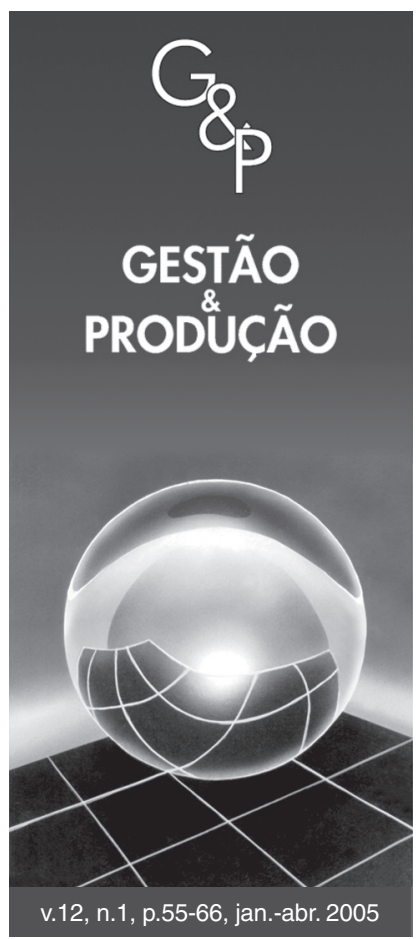

\title{
UMA PROPOSTA DE APLICAÇÃO DE BUSINESS INTELLIGENCE NO CHÃO-DE-FÁBRICA
}

\author{
Marcos Roberto Fortulan \\ Tecumseh do Brasil Ltda, \\ Rua Cel. Júlio Augusto de Oliveira Salles, 478, CEP 13570-900, São Carlos, SP, \\ e-mail: fortulan@ tecumseh.com.br
}

Eduardo Vila Gonçalves Filho

Escola de Engenharia de São Carlos, EESC, Departamento de Engenharia Mecânica, Universidade de São Paulo, Av. Dr. Carlos Botelho, 1465, Centro, CEP 13560-250, São Carlos, SP, e-mail: evila@sc.usp.br

$1^{\text {a }}$ Versão Recebida em 24/6/2003

$2^{\text {a }}$ Versão Recebida em 23/7/2004

Aceito em 25/2/2005

Resumo

A evolução do chão-de-fábrica tem sido significativa nas últimas décadas, quando grandes investimentos têm sido realizados em infra-estrutura, automação, treinamento e sistemas de informação, transformando-o numa área estratégica para as empresas. O chão-de-fábrica gera hoje grande quantidade de dados que, por estarem dispersos ou desorganizados, não são utilizados em todo o seu potencial como fonte de informação. Com vistas nessa deficiência, este trabalho propõe a implantação de um sistema de Business Intelligence por meio do uso de ferramentas de Data Warehouse e OLAP (On-Line Analytical Processing), aplicadas especificamente ao chão-de-fábrica. O objetivo é desenvolver um sistema que utilize os dados resultantes do processo produtivo e os transforme em informações que auxiliem o gerente na tomada de decisões, de forma a garantir a competitividade da empresa. Um protótipo foi construído com dados simulados para testar a proposta.

Palavras-chave: sistema de apoio à decisão, business intelligence, data warehouse, OLAP, chão-de-fábrica.

\section{Introdução}

O chão-de-fábrica, como conhecemos hoje, começou a delinear-se após a Revolução Industrial. Antes dela, a produção de bens se dava, basicamente, por meio de pequenas oficinas, que tinham como uma característica importante o fato de que o conhecimento a respeito do produto final e como fazê-lo estava associado àquele que efetivamente fabricava o produto, ou seja, ao artesão.

A busca incessante do homem por melhores condições de vida, aliada ao desenvolvimento tecnológico, gerou novas necessidades a respeito de qualidade, quantidade e preço dos produtos, o que fez a antiga oficina dar lugar ao que chamamos hoje de indústria. Nas indústrias, um conjunto de homens e máquinas passou a ocupar um mesmo espaço na chamada produção seriada, e com ela ocorreu a transferência da habilidade de fabricação do homem para a máquina. Com a indústria, o conhecimento sobre o produto final foi separado daquele que o produzia. Essa separação deu origem a uma nova classe social em subs- tituição ao artesão, o proletariado, e a uma nova área em substituição às oficinas, o chão-de-fábrica.

Hoje encontramos empresas onde o chão-de-fábrica, ao contrário de um século atrás, é organizado, limpo e seguro, fruto de grandes investimentos em infra-estrutura, automação e treinamentos. Nelas, o chão-de-fábrica exige profissionais qualificados para lidar com equipamentos complexos, buscar melhorias contínuas e com postura voltada à qualidade e satisfação do cliente, foco que não existia no início da industrialização. Dentro deste novo cenário, durante o processo produtivo é gerada uma grande quantidade de dados relacionados à qualidade, produtividade, manutenção, máquinas, materiais, produtos, funcionários, etc. No entanto, muitas empresas ainda não sabem o que fazer com essa massa de dados, desconhecendo sua importante utilidade como matéria-prima para a geração de informações úteis à gestão do negócio. Muitos gerentes de chão-de-fábrica, que hoje sofrem por falta 
de informações, podem ter um grande aliado se dispuserem de ferramentas para processar os dados.

Esses dados têm pouca utilidade em seu estado bruto, por isso precisam ser tratados e interpretados para que deles seja possível tirar informações e conhecimento. Para tal, existem hoje diversas ferramentas específicas e disponíveis comercialmente. Empresas do mundo da Tecnologia da Informação, como Oracle, IBM, Seagate e Microsoft, oferecem softwares que podem ser ajustados às necessidades de cada usuário ou corporação. Esta área vem hoje sendo tratada como Sistemas de Apoio à Decisão (SAD) ou Business Intelligence (BI), termo que vem sendo mais explorado ultimamente.

Atualmente, as principais aplicações de $B I$ são encontradas nas áreas de finanças, marketing e atendimento ao cliente, maciçamente concentradas em bancos, seguradoras e empresas de comércio. É, portanto, exatamente para essa lacuna que este trabalho está voltado, ou seja, para o tratamento dos dados do chão-de-fábrica por intermédio de uma ferramenta de $B I$.

A proposta é apresentada no decorrer deste trabalho, ao longo de mais quatro seções. A seção 2 tem como objetivo introduzir o conceito dos Sistemas de Informação e sua importância, destacando características como flexibilidade e integração, pois o $B I$ passa a ser mais um elemento deste sistema ao lado, principalmente, dos sistemas ERP (Enterprise Resource Planning). A seção 3 faz uma revisão sobre os conceitos de Business Intelligence e Sistemas de Apoio à Decisão, destacando suas ferramentas mais recentes: Data Warehouse, OLAP (OnLine Analytical Processing), Data Mining e Web-Business Intelligence. Na seção 4 é discutida a proposta do BI especificamente para o chão-de-fábrica, é sugerido um modelo dimensional de banco de dados e são detalhadas suas tabelas de Fato. Também são apresentadas na seção 4 a capacidade do sistema, as limitações e decisões que devem ser tomadas antes de se iniciar o projeto. Por fim, na seção 5, faz-se uma conclusão utilizando-se uma situação hipotética para discutir a viabilidade e ganhos potenciais da proposta.

\section{Sistemas de gestão da informação}

Todas as empresas têm enfrentado nos últimos anos um grande aumento na quantidade de informações dentro e fora delas, seja para controlar seus processos internos - manufatura, contabilidade, custos, logística, comercial, projeto - seja para relacionar-se com a sociedade, clientes e fornecedores. Toda esta estrutura é suportada pelos sistemas de informações existentes na organização, que guiam todas as atividades da empresa e, por isso, pode-se dizer que o sucesso de uma organização está estreitamente ligado ao sucesso do seu Sistema de Informações (SI).

O papel do SI sofreu uma mudança dramática ao longo das últimas três décadas, passando de uma passiva fer- ramenta de automação ou argumentação, para um dispositivo estratégico e competitivo de transformação das estruturas organizacionais (Lee e Kim apud Avison et al., 1999, p. 421). Hoje é imprescindível que esse sistema seja flexível e integrado, para que se possibilite editar ou incluir novos processos sem comprometer o desempenho daquilo que já está em funcionamento, no qual todas as áreas da organização estejam envolvidas, canalizando as informações essenciais para todos os níveis que delas necessitem, levando todos à otimização. É necessário, portanto, que, ao incluir um novo componente no SI, este seja flexível e se integre com os demais componentes, o que vale para os $B I$.

Não é possível citar um SI ideal, geral ou único para qualquer atividade que seja, pois, além da enorme quantidade de soluções disponíveis comercialmente, os desenvolvimentos não param, trazendo novos recursos a cada dia. No entanto, a integração é um ponto em comum entre todas as ferramentas que buscam colocar dentro de um único sistema todas as informações relativas a todas as áreas e unidades da empresa, seus clientes, seus fornecedores, inclusive a Web.

A seguir, na Figura 1, é apresentado um SI genérico, com o objetivo de mostrar o fluxo dos dados e informações. Pela figura, observa-se que o ERP alimenta os bancos de dados operacionais, que por sua vez servirão de fonte de dados para o Data Warehouse. No entanto, antes de serem colocados para dentro do Data Warehouse, os dados devem ser tratados e processados. Uma vez povoado o Data Warehouse, podem-se aplicar ferramentas OLAP e Data Mining para a obtenção de informações e, assim, teremos o ambiente de $B I$. Nesta figura, embora não seja mencionada a integração com a $W e b$, vale ressaltar que a Web já está integrada a tais SI, como será abordado na seção 3, no assunto Web Business Intelligen$c e$. A seguir, é apresentado o conceito de ERP e, posteriormente, na seção 3 deste trabalho, serão discutidos os conceitos de Data Warehouse e Data Mining.

Os ERP (Enterprise Resources Planning) são uma evolução dos sistemas MRP (Manufacturing Requirements Planning) e MRP II (Manufacturing Resources Planning), dos quais um dos objetivos é consolidar as informações da empresa como um todo, agregando as funções de Planejamento e Controle da Produção, Planejamento das Necessidades de Materiais, Cálculo da Capacidade de Produção, Controle do Chão-de-Fábrica, Controle de Compras, Gerenciamento de Recursos Humanos, Vendas e Distribuição, Finanças e Controladoria, entre outros, dentro de um único sistema, eliminando assim a dificuldade de se obter informações consolidadas e a inconsistência de dados redundantes armazenados em mais de um sistema (Zancul e Rozenfeld, 1999).

A importância do ERP neste trabalho, como já citado, é considerar que ele será a fonte primária dos dados que 


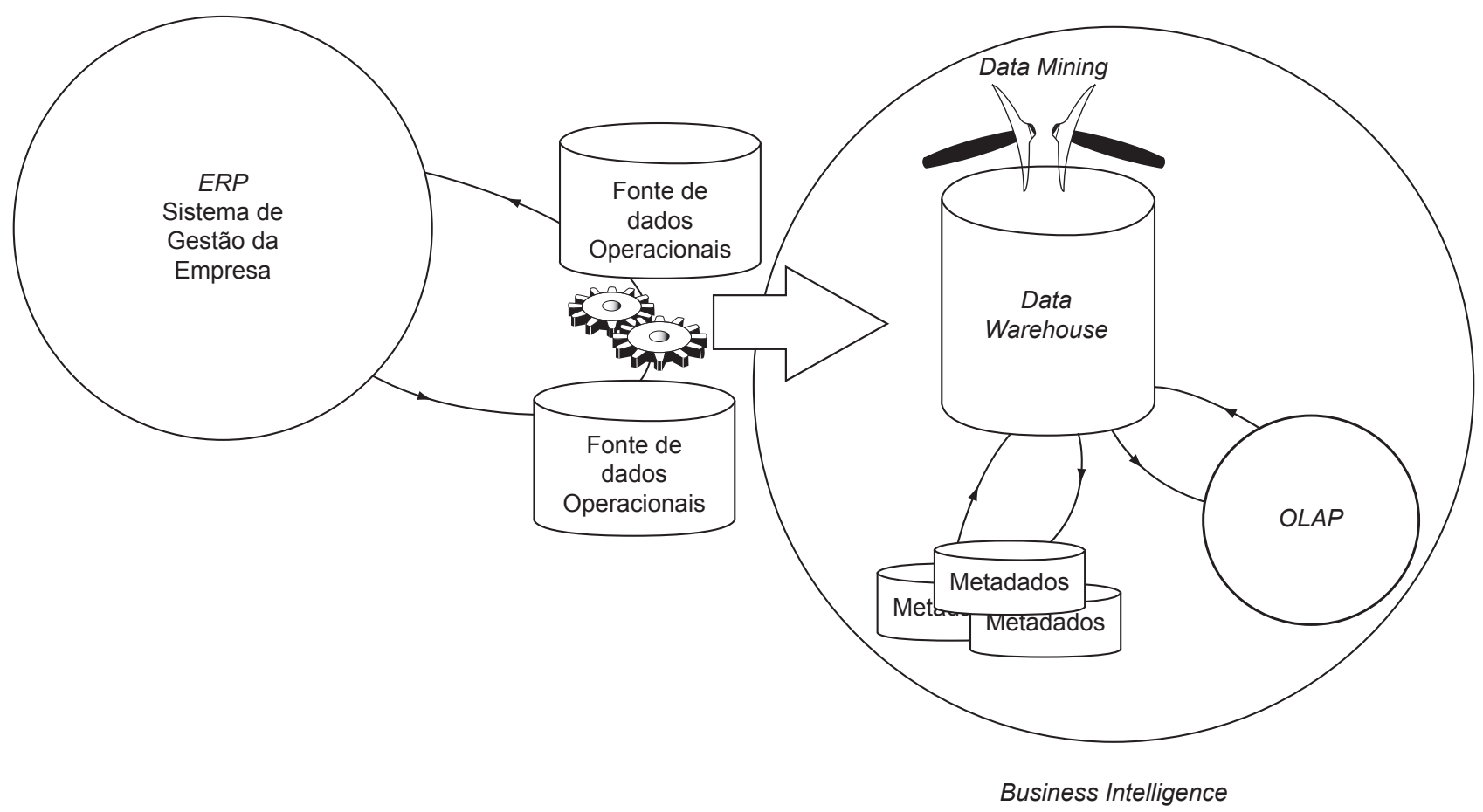

Figura 1. SI integrado e o fluxo de dados e informações.

alimentarão o Data Warehouse, levando-se em conta que este é um sistema já comum em empresas de grande porte ou de classe mundial.

\section{Os novos Sistemas de Apoio à Decisão (SAD) ou Business Intelligence (BI)}

Para competir no mercado global de hoje, as empresas precisam deter mais conhecimento do que antigamente e, ainda, para obter sucesso, elas precisam saber mais sobre seus clientes, mercados, tecnologias e processos, e precisam ter essas informações antes que seus concorrentes (Heinrichs e Lim, 2003). Ter informações em mãos é uma poderosa ferramenta para quem precisa tomar decisões e, tendo em vista esse princípio, as empresas começaram a extrair dados dos seus sistemas operacionais e armazená-los, separados dos dados operacionais, donde surgiram os SAD.

Shim et al. (2002) dizem que os SAD são soluções computacionais desenvolvidas para apoiar a tomada de decisões complexas durante a resolução de problemas. Ferramentas clássicas de SAD compreendem componentes para gerenciamento de sofisticados bancos de dados, poderosas funções de modelagem e poderosos, embora simples, projetos de interface com o usuário, que permitem trabalhar interativamente com questões, relatórios e funções gráficas.

Carlsson e Turban (2002) explicam que o termo SAD, propriamente dito, tem sido visto cada vez menos, tanto em revistas quanto em Websites de vendas, e no seu lugar tem sido cada vez mais freqüente o uso de termos como Business Intelligence e OLAP. Do mesmo modo, estes termos praticamente eliminaram o uso do termo EIS (Executive Information Systems). Por outro lado, está crescendo o reconhecimento de que $B I$ está se tornando um componente necessário na chamada segunda geração dos sistemas ERP, que claramente reconhece a necessidade de dar suporte não apenas ao processamento de transações operacionais, mas também ao processamento de análises.

Complementam Grigori et al. (2004) que com dados limpos e agregados sobre um determinado processo, armazenados em um Data Warehouse, é possível realizar análises utilizando-se tecnologias de $B I$ e extrair conhecimento sobre as circunstâncias que levaram a determinado resultado no passado, tenha o resultado sido bom ou ruim. Assim, é possível utilizar essas informações para explicar por que tais circunstâncias ocorreram e para predizer potenciais problemas nos processos em andamento.

Os Data Warehouses, OLAP, Data Mining e WebSAD surgiram no começo dos anos 90 como novas ferramentas para $\mathrm{SAD}$, e formam a base dos sistemas de $B I$ (Shim et al., 2002). Estas ferramentas serão discutidas na sequiência.

\subsection{Data Warehouse}

Ao longo do tempo, os bancos de dados foram desenvolvidos para fins de processamentos de dados operacionais e analíticos, havendo maior ênfase no primeiro caso, ainda que ambos tivessem usuários com diferentes necessidades. Uma vez compreendida essa diferença, foram criados bancos de dados separados para fins ana- 
líticos, chamados de Data Warehouse, explicam Gray e Watson (1999). É inevitável a perda de produtividade e confiabilidade quando os dados são retirados de diversas fontes em sistemas desintegrados; assim, o conceito de Data Warehouse propicia integração e consolidação da informação.

Conforme Harding e Yu (1999), o Data Warehouse extrai, de uma maneira eficiente, informações valiosas dos bancos de dados operacionais que estão espalhados por toda a empresa e que, certamente, contêm dados duplicados ou parcialmente duplicados. Por isso, antes de povoar o Data Warehouse com os dados operacionais, estes dados precisam ser preparados para estarem aptos a serem questionados, pesquisados, analisados e apresentarem conclusões. Nas aplicações de $B I$, o foco está mais voltado para as tendências e agregações do que para cada transação individual; portanto, os dados armazenados devem oferecer níveis de agregação apropriados.

Gray e Watson (1999) destacam como características destes bancos:

- São orientados a determinado assunto;

- São integrados;

- Não são violáveis, ou seja, seus dados não são alterados, só incluídos;

- Representam um histórico;

- São condensados;

- Representam um longo período de tempo (de 5 a 10 anos);

- Não são normalizados, ou seja, podem ter dados redundantes;

- Não são baratos e têm implantação demorada; e

- São grandes e continuam crescendo ao longo do tempo.

Para o desenvolvimento de um Data Warehouse, é utilizada a modelagem dimensional de banco de dados, cuja representação se dá por meio do chamado esquema Estrela. Algumas vantagens deste modelo de banco de dados sobre os mode los relacionais convencionais para aplicações de Data Warehouse são citadas por Poe et al. (1998) e Bispo (1998) e descritas abaixo:

- Permite a criação de um projeto de banco de dados que fornecerá respostas rápidas, com menos tabelas e índices;

- Permite ao administrador do banco de dados trabalhar com projetos mais simples e assim produzir melhores planos de execução; e

- Possui uma estrutura mais intuitiva, assemelhando o projeto do banco de dados com a forma como o usuário final pensa e usa os dados.

O modelo dimensional é composto basicamente por dois tipos de tabelas: as de Fato e as de Dimensão. As primeiras são grandes tabelas centrais, compostas basica- mente das ocorrências do negócio, por exemplo, vendas, produção e defeitos. Já as segundas armazenam as descrições do negócio, como dados sobre o produto (marca, tamanho, categoria, preço), tempo (dia, mês, ano) ou cliente (nome, endereço, classe social). Cada tabela de Dimensão possui uma única chave primária, e o conjunto dessas chaves primárias formará a chave composta da tabela de Fato. Cada negócio ou assunto possui sua tabela de Fato e suas respectivas tabelas de Dimensão.

Uma variação do esquema Estrela é o Snowflake, que consiste, na realidade, de uma normalização do primeiro. No esquema Snowflake, as tabelas de Dimensão são estruturadas de modo que atendam à terceira forma normal, mantendo as tabelas de Fato em sua estrutura inicial. Vale ressaltar que o uso do esquema Snowflake traz como desvantagens o aumento da complexidade da estrutura de dados, dificultando a compreensão do modelo por parte de usuários que trabalham diretamente com a estrutura física das tabelas. No entanto, o uso do Snowflake pode ser indispensável em alguns casos em que, por exemplo, o modelo desnormalizado (Estrela) requeira muito espaço em disco ou suas tabelas dimensionais sejam muito grandes, prejudicando o desempenho do sistema (Singh, 2001 e Poe et al., 1998). A Figura 2 mostra o formato geral de um esquema Estrela e uma derivação Snowflake.

Muito utilizados em projetos-piloto, existem também os Data Marts, que, conforme Singh (2001) define, são subconjuntos do Data Warehouse da empresa-inteira. Tipicamente desempenham o papel de um Data Warehouse departamental, regional ou funcional. Uma empresa pode construir uma série de Data Marts ao longo do tempo e, eventualmente, vinculá-los por meio de um Data Warehouse lógico da empresa-inteira.

\subsection{On-Line Analytical Processing (OLAP)}

Bispo e Cazarini (1998) apresentam o OLAP como uma ferramenta capaz de efetuar análises de dados com visão multidimensional do negócio, comparando-os por diversos ângulos. Aplicações que utilizam este tipo de ferramenta devem ter como características:

- Permitir visão multidimensional dos dados;

- Realizar cálculos complexos;

- Criar agregações e consolidações;

- Fazer previsões e análise de tendência;

- Construir cenários a partir de suposições; e

- Fazer cálculos e manipular dados através de diferentes dimensões.

Os cubos são os principais objetos de um OLAP. Construídos com tecnologia que permite rápido acesso aos dados, normalmente eles são construídos a partir de sub-conjuntos de um Data Warehouse e são organizados e sumarizados dentro de estruturas multidimensionais defi- 


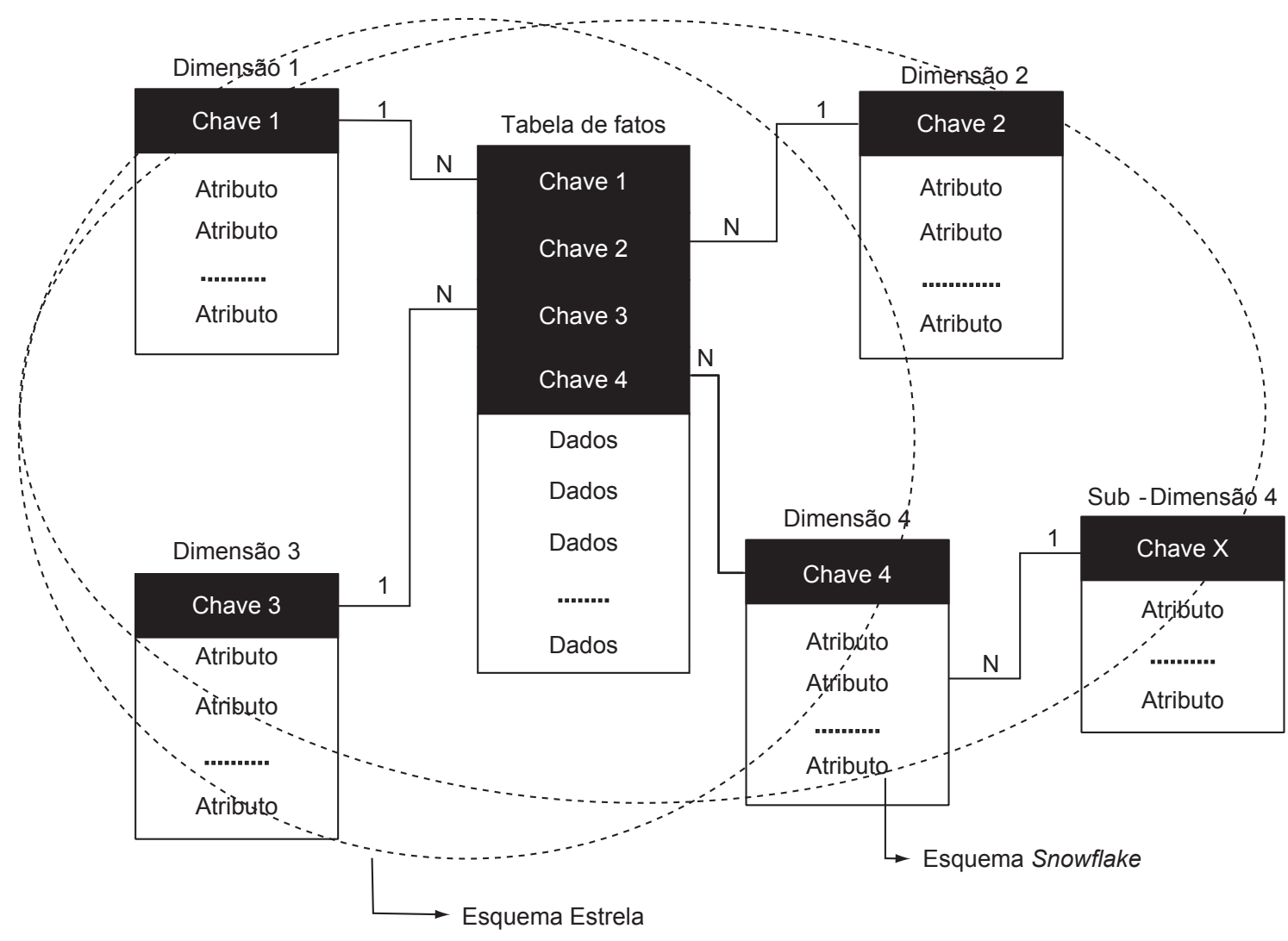

Figura 2. Modelo Dimensional de banco de dados utilizando esquema Estrela e Snowflake.

nidas por dimensões e medidas. Os bancos de dados multidimensionais guardam as informações numa espécie de cubo de dimensão "n", o que resulta em diversas matrizes esparsas que permitem trabalhar simultaneamente com diversos cenários definidos por combinações de dados, como produtos, região, vendas, tempo, etc. Esses cubos podem ser armazenados em modelos de bancos de dados ROLAP (Relacional OLAP), MOLAP (Multidimensional $O L A P$ ) ou HOLAP (Híbrido OLAP). A vantagem dos bancos de dados multidimensionais sobre os bancos de dados relacionais é que os primeiros estão otimizados para obter velocidade e facilidade de resposta, complementam Gray e Watson (1999).

\subsection{Data Mining}

Conforme Bispo e Cazarini (1998), o objetivo desta ferramenta é extrair informações potencialmente úteis a partir de dados "crus" armazenados em um Data Warehouse ou em bancos de dados dos sistemas operacionais, porém, preferencialmente nos primeiros, por já estarem consolidados. Para tal, utilizam-se técnicas sofisticadas de procura, como algoritmos de Inteligência Artificial, Redes Neurais, Árvores de Decisões, Regras de Indução, ou ainda, combinações entre elas.

O resultado obtido pela aplicação do Data Mining deve ser compacto, legível (apresentado de alguma forma simbólica), interpretável e deve representar fielmente os dados que lhe deram origem. Isso é necessário porque as pessoas normalmente estão mais interessadas em obter o conhecimento propriamente dito do que os modelos matemáticos, afirma Bäck (2002).

\subsection{Web SAD ou Web Business Intelligence}

Tan et al. (2003) e Henrichs e Lim (2003) destacam o recente desenvolvimento e crescimento do que pode ser chamado de Web Business Intelligence (WBI). WBI mescla Data Warehousing e sistemas de BI com as novas tecnologias da Web. A Internet, associada com as tecnologias da $W e b$, tem mudado a forma como as pessoas fazem seus negócios. A Web é um modelo de sucesso, e seu ambiente distribuído e os Web browsers oferecem excelentes exemplos de como dispor software e acesso de dados dentro das organizações. As vantagens do uso de sistemas de $B I$ baseados na Web, quando comparados com os sistemas tradicionais, incluem facilidades de uso, acesso universal às diversas plataformas, possibilidade de configuração para diversos níveis de usuários e capacidade de trabalho com gráficos, sons e vídeos além de textos e números.

Os Web-BI têm reduzido barreiras tecnológicas, tornando mais fácil e barato disponibilizar informações relevantes para tomadas de decisão aos gerentes da cor- 
poração, mesmo que estes estejam geograficamente em localizações diferentes. Construir Web-BI é uma tarefa complexa, pois deve considerar um sistema que esteja disponível sete dias por semana, vinte e quatro horas por dia e deve manipular um grande número de solicitações concorrentes (Shim et al., 2002).

O modelo proposto na seção 4 não aborda sua aplicação na Web, porém a ferramenta utilizada nele, o Analysis Services do Microsoft SQL Server 2000, já está preparada para esta integração, tratando-se apenas de uma questão técnica.

\section{Aplicação do BI no chão-de-fábrica - modelo proposto}

Os dados gerados no chão-de-fábrica normalmente ficam espalhados pelos diversos bancos de dados operacionais ou documentos em papel da área, forma de armazenagem que dificulta em muito a recuperação dos dados e o levantamento de comportamentos históricos. Essa é uma situação crítica quando se trata de programas de melhorias, como os conduzidos por Seis Sigma, FMEA (Failure Mode and Effects Analysis ou Análise do Modo e Efeito da Falha) ou PDCA (Plan, Do, Check and Action ou Planejar, Executar, Verificar e Agir). Esses programas podem perder sua força por causa da dificuldade de se obter informações sobre condições passadas e futuras em relação às suas implementações. Isso dificulta e, às vezes, até impossibilita a análise da eficácia das mudanças e dos fatores relacionados a ela. Este fato acaba prejudicando o processo de mudança em si, pois não são conhecidos os benefícios ou prejuízos trazidos pelas alterações. É uma situação que pode ser melhorada com a implantação do modelo proposto, que aplica o $B I$ no chão-de-fábrica.

Outra grande vantagem dos sistemas de $B I$ é sua facilidade para trabalhar com questões ad-hoc, pois é praticamente impossível desenvolver uma aplicação em que todos os relatórios e pesquisas de que um gerente possa precisar para realizar seu trabalho estejam presentes. Já os sistemas de $B I$ permitem criar facilmente relatórios e pesquisas específicas relativas às necessidades emergentes de cada gerente ou momento.

\subsection{Trabalhando com bancos de dados do Data Warehouse ou do OLTP (On line Transactions Processing)}

A proposta de desenvolvimento de um Data Warehouse pode assustar num primeiro instante, dada sua complexidade, custos e longo prazo de desenvolvimento. Realmente, antes de partir para a adoção de qualquer ferramenta, é importante saber o que se deseja. O foco deve estar voltado para as informações desejadas, e não para a tecnologia que será utilizada para obtê-las. Se houver dúvidas quanto à relação Custo $\mathrm{X}$ Benefício do projeto, talvez seja aconselhável melhorar os sistemas OLTP $(O n$ line Transactions Processing) já existentes na empresa, e nestes aplicar ferramentas convencionais para a geração de relatórios, como o Crystal Reports, Excel ou mesmo ferramentas $O L A P$, enquanto se analisam melhor as soluções baseadas em Data Warehouses. As opções são diversas e devem ser pensadas antes de se iniciar o projeto. A principal vantagem dos Data Warehouses é que eles são bancos de dados projetados exclusivamente para a geração de relatórios, pois possuem propriedades específicas para a análise de dados. Outra vantagem é a liberação dos sistemas $O L T P$ para a realização exclusiva das transações operacionais, que normalmente têm que ser executadas on-line e freqüentemente tratam diretamente com o cliente, que, entre outras características, não gosta de esperar. Agora, uma vez que se decide pela construção de um sistema exclusivo para apoio da tomada de decisão, os Data Warehouses, com certeza, são o ponto final desta procura.

Pode-se ainda, antes de partir para o desenvolvimento de um Data Warehouse corporativo, trabalhar com Data Marts, que são subconjuntos de um Data Warehouse voltados para uma determinada função, assunto ou departamento em particular, que no presente artigo é o chão-defábrica. Os Data Marts são menores e de implantação mais rápida, e podem ser tratados inclusive como um projeto piloto para que a empresa comece a conhecer os benefícios desta tecnologia. Assim, neste projeto, os dados do chão-de-fábrica irão compor uma parte dos dados do Data Warehouse, ou seja, teremos um Data Mart.

Deve-se, no entanto, tomar cuidado com a proliferação dos Data Marts sem um sistema que os consolide, entenda-se aqui um Data Warehouse, pois estes sistemas rapidamente estarão desintegrados e, junto dessa desintegração, trarão todas as conseqüências já citadas. Não há problemas em se começar pequeno, mas não se pode perder a visão do sistema maior, o Data Warehouse corporativo, reforça Corey et al. (2001). O Data Warehouse normalmente se inicia nas áreas de marketing, vendas e finanças, pois nestas fica mais fácil justificar o investimento, uma vez que elas estão diretamente relacionadas com as transações financeiras ou com os clientes da empresa.

\subsection{Desenvolvimento do sistema no chão- de-fábrica}

Neste trabalho, o modelo proposto de $B I$ é composto de cinco tabelas de Fato e onze tabelas de Dimensão, estando cada tabela de Fato relacionada a um comportamento do cotidiano de uma área típica de chão-de-fábrica. A escolha dos assuntos que compõem as tabelas de Fato teve como intenção produzir informações a respeito daquilo que mais interessa ao cliente, que são custo, qualidade e prazo. Assim, as cinco tabelas de Fato propostas 
têm como incumbência representar o custo, a qualidade e o prazo do chão-de-fábrica, o que, é claro, será sentido pelo cliente. Neste trabalho, quando se fala em custo, deve-se entendê-lo como uma função direta da eficiência e qualidade da área, pois não será tratado neste Data Mart o valor financeiro dos produtos ou processo. Já o prazo está relacionado à eficácia do atendimento das datas de fabricação.

Para a realização dos testes deste modelo, foi construído um Data Mart utilizando-se o banco de dados Microsoft Access e o Analysis Services, software componente do Microsoft SQL Server 2000. Os exemplos de análise aqui apresentados foram realizados sobre o Data Mart de Desempenho, como pode ser visto na Figura 3. Entenda-se como desempenho, neste trabalho, a quantidade produzida, a eficiência e a eficácia, sumarizadas por data, célula, equipe, turno e produto. Nesta tabela, a eficiência é relativa ao aproveitamento da mão-de-obra, e a eficácia é relativa ao cumprimento dos prazos de entrega previstos nas ordens de fabricação.

A tabela Fato de Desempenho foi povoada com dados simulados, procurando atingir uma situação real baseada na experiência dos autores. Sua finalidade é permitir analisar a funcionalidade da proposta. A partir deste Data Mart, foram construídos dois cubos, sendo um para Produção e outro para Eficiência. A flexibilidade e facilidade das pesquisas que podem ser realizadas pelo Analysis Services são bastante amplas, possibilitando uma ferramenta realmente útil para a tomada de decisão do gerente da área. Por meio da Figura 4 podemos obter, por exemplo, as seguintes informações: i. A unidade brasileira do grupo produz 2,79 vezes mais que a americana;

ii. No Brasil, por sua vez, a unidade do Rio de Janeiro é maior, pelo menos em produção, que a de São Paulo; e

iii.Dentre as áreas brasileiras, a Área D é a que está sendo mais utilizada, enquanto a Área B é a que está, teoricamente, mais ociosa.

Combinações entre as dimensões produto, equipe, turno e tempo também podem ser realizadas facilmente dentro deste cubo, bem como operações de drill-down, drill-up, slice and dice. Com base nessas informações, o gerente pode decidir por redistribuir sua capacidade de produção entre suas unidades, áreas, turnos e equipes, considerando-se, por exemplo, a demanda existente em cada localidade.

O segundo cubo montado neste Data Mart é o cubo de Eficiência. Com ele é possível responder à seguinte pergunta: Qual o turno que tem maior e qual o que tem menor eficiência? Com base no cubo apresentado na Figura 5, é possível verificar que, de forma geral, o turno "A" possui menor eficiência, e o turno " $C$ ", maior. No entanto, analisando-se por país, esta afirmação nem sempre é verdadeira. Por isso, é necessário estar capacitado a analisar as informações geradas no sistema. Elas podem requerer questionamentos mais aprofundados antes de se tirar uma conclusão sobre a situação. Isso pode estar acontecendo, por exemplo, por que em um país existe maior resistência para a aceitação de determinado horário de que no outro. Podem estar acontecendo também problemas específicos

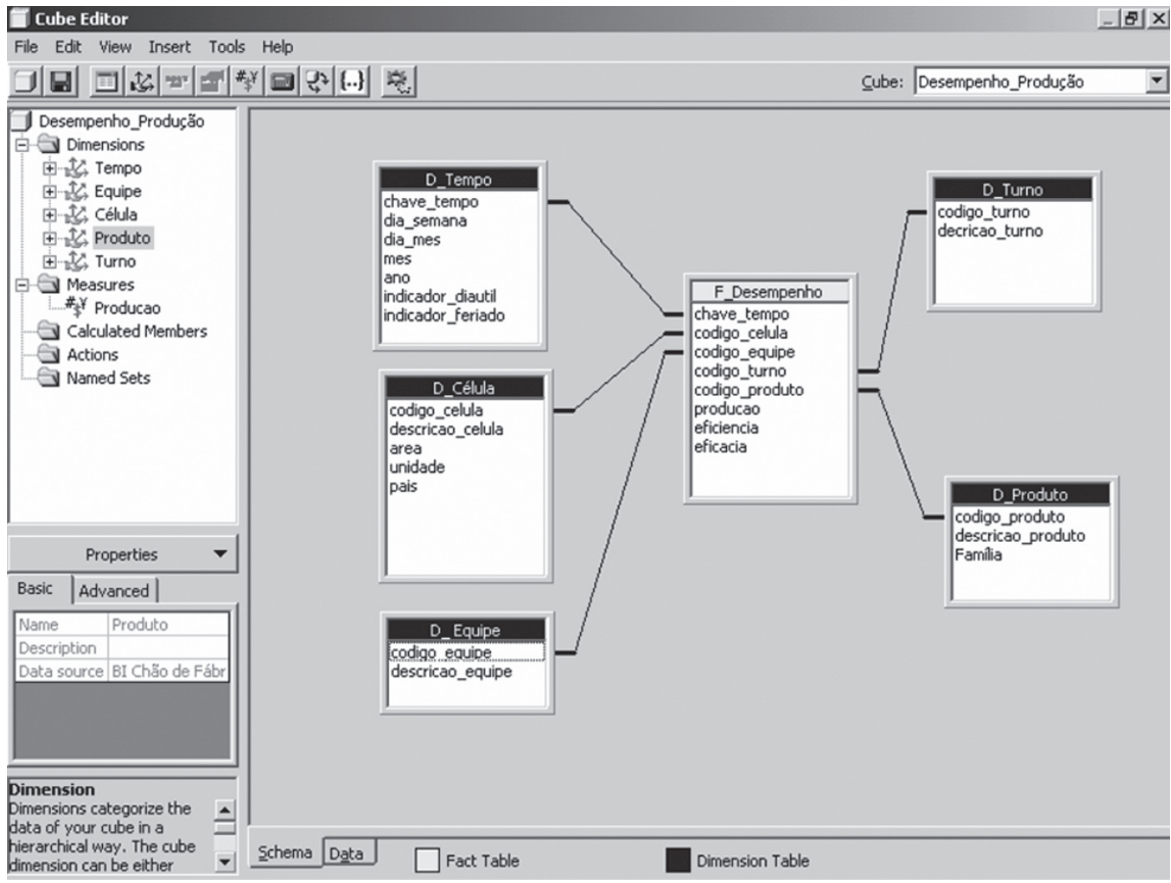

Figura 3. Data Mart Desempenho modelado no Analysis Services. 


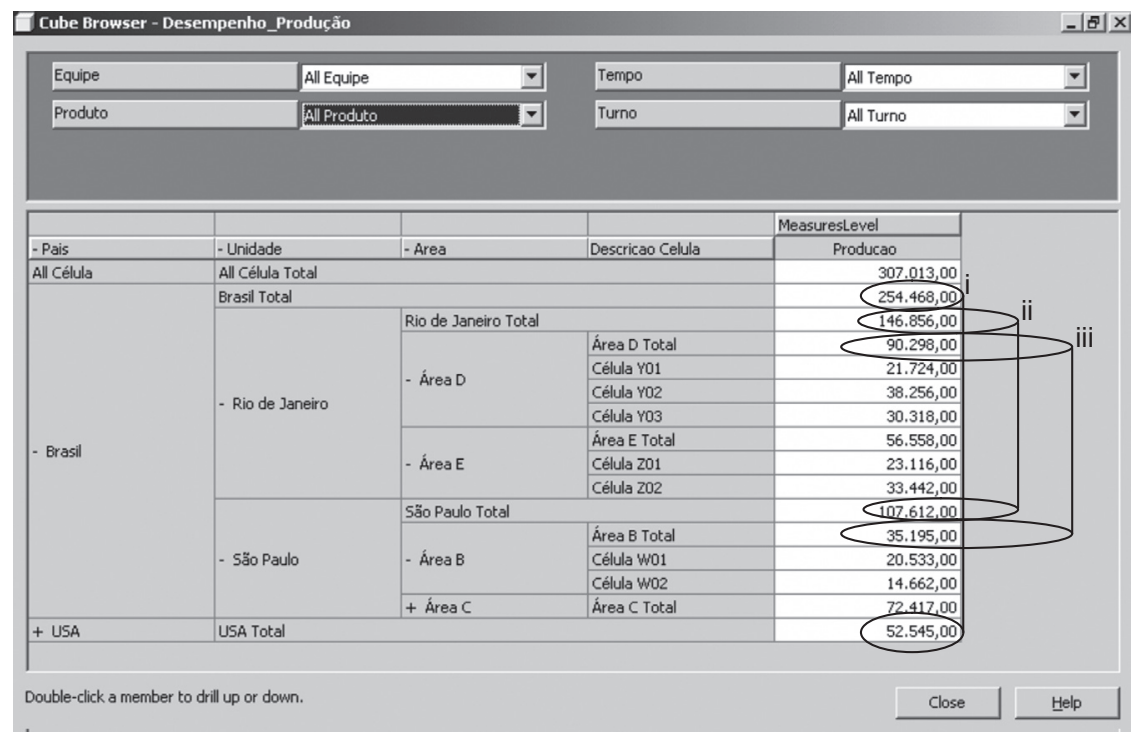

Figura 4. Cubo de Produção construído no Analysis Services.

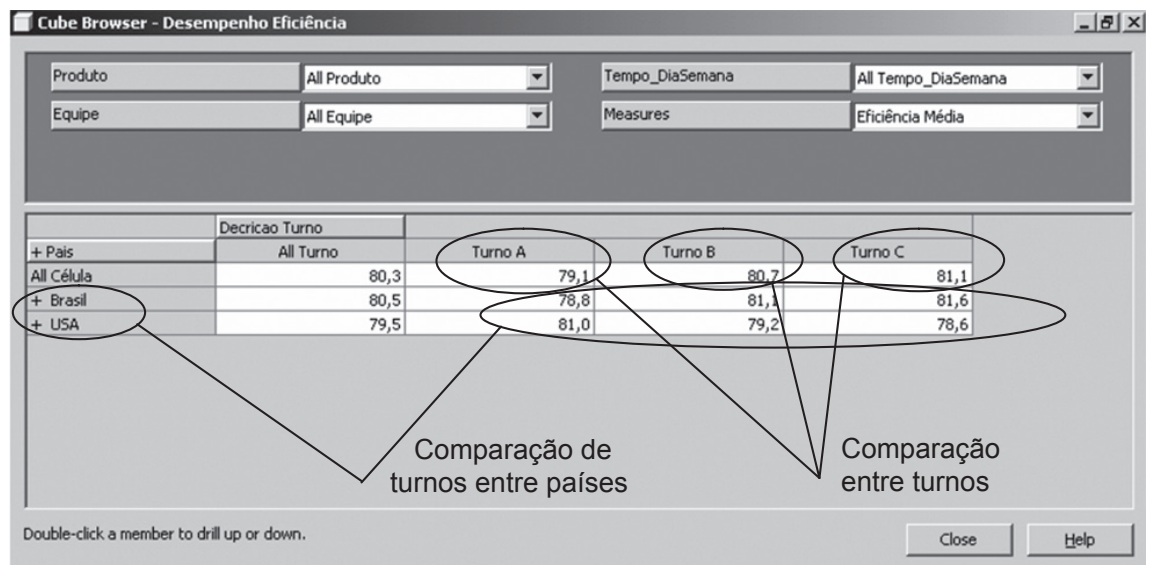

Figura 5. Cubo de Eficiência com foco no turno e célula.

em determinado turno, como alteração da temperatura ambiente. Com base nessas informações, o gerente deve pensar em ações que minimizem seus efeitos e revertam num ganho de desempenho. Ou seja, o sistema não dá a resposta para o problema, mas sim gera informações sobre comportamentos da área, que precisarão ser analisados por pessoal competente e com poder de ação. Por isso, é chamado de um sistema de apoio às decisões, requerendo a presença de um tomador de decisão, no caso aqui, o gerente do chão-de-fábrica.

Dentro deste mesmo cubo, é possível ainda verificar o que acontece com a eficiência das equipes e ver se há alguma variação quando ocorre a mudança de turnos, como mostrado na Figura 6, ou também observar se há variação da eficiência em função do dia da semana, como aparece na Figura 7. Em ambos os casos, caberá uma atitude gerencial para verificar e resolver o porquê de as eficiências das equipes caírem quando as equipes trabalham no "Turno A" (Figura 6) ou o que acontece durante determinado dia da semana, quando a eficiência é menor (a Figura 7 mostra que neste exemplo esta variação não é significativa). Deve-se tomar cuidado para não atribuir a causa a uma determinada variável e penalizá-la quando o problema pode estar em outra, porém o fato é que existe um problema. Análises semelhantes podem ser feitas também levando-se em conta o produto ou família de produtos que estão sendo fabricados. Por fim, de posse do Data Mart povoado, é possível construir cubos geradores de informações que permitirão ao gerente direcionar suas ações de forma a melhorar o desempenho de sua área e, também, acompanhar o resultado de suas ações.

No entanto, conforme a proposta inicial, o $B I$ para o chão-de-fábrica é composto por cinco tabelas de Fato, pois visa possibilitar uma análise completa do comporta- 


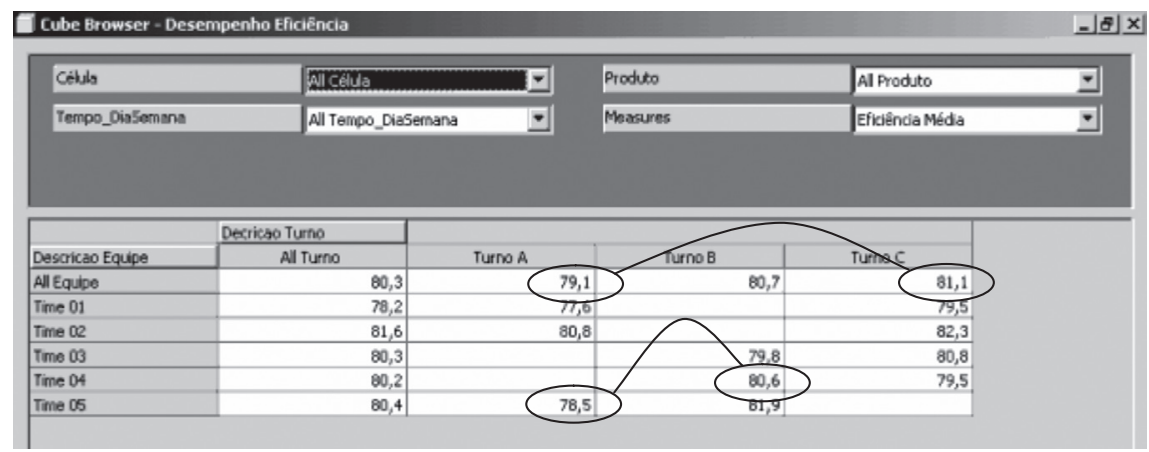

Figura 6. Cubo de Eficiência com foco no turno e equipe.

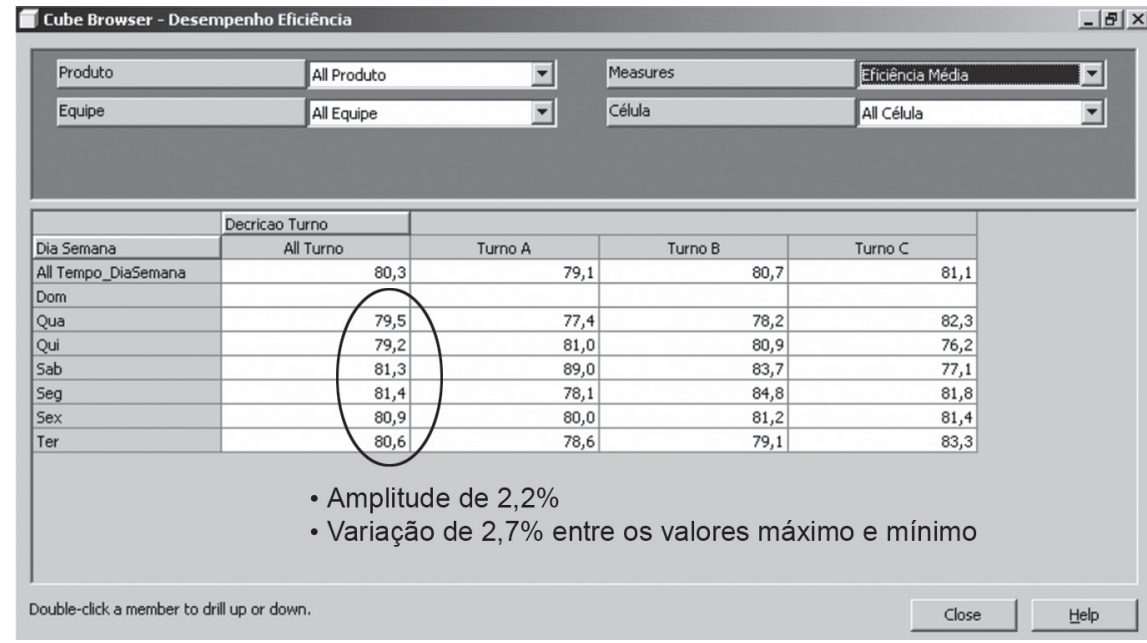

Figura 7. Cubo de Eficiência com foco no dia da semana e turno.

mento da área. Embora os Data Marts e cubos do modelo completo não tenham sido mostrados aqui neste trabalho, tal modelo já foi implementado e testado no Analysis Services com resultados de desempenho iguais aos obtidos pelo Data Mart Desempenho.

As demais tabelas de Fato que compõem o modelo completo são: Medições, Qualidade, Horas-Perda e Evento. A tabela Medições tem o intuito de verificar a conformidade do processo produtivo, e nela são registrados os valores de medições realizadas no processo produtivo, sumarizadas por dia de trabalho, máquina, produto e processo de medição. Cada processo de medição da empresa deve ser identificado por meio de um único código que sirva para toda a empresa ou corporação.

A partir do Data Mart montado sobre esta tabela, aplicando-se o Analysis Services, podemos obter respostas para perguntas do tipo:

- Qual o comportamento da média, desvio-padrão, máximo e mínimo das medidas de "Rugosidade" do produto "A", fabricados no ano de 2004 na "Máquina AA", ao longo dos meses mês a mês? Este tipo de informação é fundamental para a condução de projetos de Seis Sigma, PDCA e FMEA.

Na tabela de Fato de Qualidade serão registrados os dados dos defeitos e sucatas detectados no processo produtivo, normalmente obtidos pelos testes ou inspeções $100 \%$. Neste caso não se trata de valores variáveis, mas sim de atributos, por exemplo, oxidação, trinca, queima, risco, não liga, etc. Os valores apontados são de quantidade de defeitos e defeitos por milhão (PPM), sumarizados por data, célula, equipe, turno, máquina, produto e tipo de defeito. A partir desta tabela, é possível verificar qual célula, equipe, turno, máquina ou produto geram mais defeitos e quais foram os tipos de defeitos. Pode-se, ainda, montar um cubo que faça uma combinação entre as várias dimensões citadas e mais as datas de fabricação.

A quarta tabela de Fato é a tabela de Horas-Perda. Nela devem ser apontadas todas as perdas de horas de produção ocorridas por causa de paradas, como manutenções e ajustes em máquinas e equipamentos. Além do código da horaperda, que deve ser único para toda a empresa, nesta tabela também é apontado o tempo perdido. A partir dela, pode-se 
descobrir qual célula, equipe, turno, máquina ou produto geram mais horas-perda, e que tipos de perda. Isso permitirá ao gerente saber para onde deve direcionar seus esforços.

Por fim, a última tabela de Fato deste modelo é a tabela de Evento. A intenção dela é registrar todos os eventos que possam afetar os resultados de Medições, Qualidade, Desempenho ou Horas-Perda. Aqui é feita uma alusão à metodologia de identificação de causa-efeito conhecida como $6 \mathrm{M}$, a saber: máquina, material, meio ambiente, método, medida e mão-de-obra. Assim, eventos observados como a introdução de um novo processo/máquina/material, ou o uso de um material suspeito, ou a introdução de equipes novas, ou a reforma de máquinas, ou a ocorrência de treinamentos, etc., deverão ser registrados. Essa prática visa sistematizar a forma de identificação e registro dos eventos, devendo estes ser apontados sempre pelo líder da equipe a cada ocorrência. A partir daí, será possível relacioná-los às demais tabelas de Fato do modelo.

A Figura 8 mostra o Data Mart completo para o chãode-fábrica, o qual, conforme já citado, foi desenvolvido com o banco de dados Access, tirando-se proveito do seu potencial de relacionamentos e edição dos bancos de dados. No entanto, o ideal para uma implementação numa situação real é a utilização de bancos de dados mais robustos para aplicações corporativas, como o Microsoft SQL Server ou o Oracle.

Para se ter uma idéia da possibilidade de consultas deste modelo, basta calcular quantas combinações de consultas são possíveis de se efetuar a partir de cada tabela de Fato em função de seus atributos e valores medidos:

- Tabela de Medições: 4 atributos e um valor.

Cmedições $=C_{4,1}+C_{4,2}+C_{4,3}+C_{4,4}=15$

- Tabela de Qualidade: 7 atributos e dois valores.

Cqualidade $=C_{7,1}+C_{7,2}+C_{7,3}+\ldots .+C_{7,7}=127 X=254$

- Tabela de Desempenho: 5 atributos e três valores.

CDesempenho $=C_{5,1}+C_{5,2}+C_{5,3}+C_{5,4}+C_{5,5}=31 \mathrm{X} 3$ $=93$

- Tabela de Horas-Perda: 7 atributos e um valor.

CHorasPerda $=C_{7,1}+C_{7,2}+C_{7,3}+\ldots .+C_{7,7}=127$

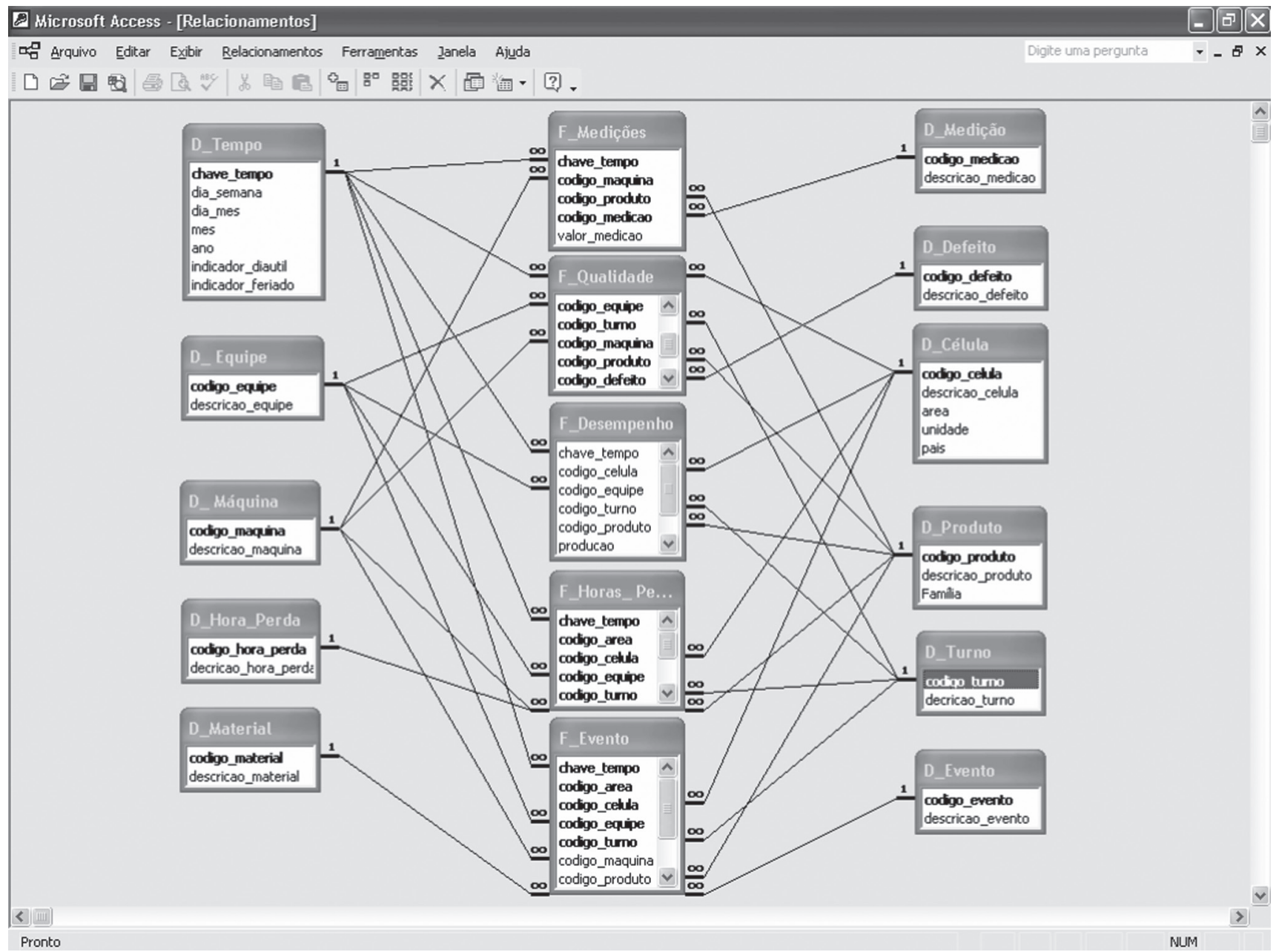

Figura 8. Modelo Estrela completo do Data Mart para o chão-de-fábrica. 
- Total = 489 combinações possíveis, isto sem considerar os possíveis subníveis de cada dimensão.

\section{Conclusão}

O chão-de-fábrica gera uma grande quantidade de dados no seu dia-a-dia, porém, eles, em grande parte das empresas, ficam espalhados em diversos bancos de dados operacionais ou em relatórios em papel, o que já dificulta sua recuperação histórica, quanto mais a obtenção de informações $a d-h o c$ a partir deles. Como exemplo, imagine uma empresa que produza 1000 peças/hora distribuídas em 10 tipos de produtos diferentes, realize inspeções em 10 células com 5 postos de inspeção cada, trabalhe em 2 turnos de 8 horas e atinja uma rejeição interna de 1000 PPM. Ela produzirá em torno de 7056 registros por mês no chão-de-fábrica, conforme mostram as contas abaixo:

- Medições $=1$ (medição) $X 10$ (células) X 5 (postos) $X$ 2 (turnos) $=100$

- Qualidade $=1000(\mathrm{pç} / \mathrm{h}) \times 16$ (horas por dia) X 1000 $\mathrm{PPM}=16$

- Desempenho $=10$ (células) X 2 (turnos) $\times 10$ (produtos) $=200$

- Eventos $=1$ (evento significativo) $X 10$ (células) $\mathrm{X}$ 2 (turnos) $=20$

- Total de registros por dia $=336$

* Total no mês (21 dias úteis) $=7056$
Com tal volume de dados, fica praticamente impossível para o gerente obter informações relevantes e em tempo hábil, se não estiver disponível uma ferramenta que sistematize e organize a recuperação dos dados. É justamente para este foco que está voltado este artigo, que propõe a construção de um sistema de $B I$ no chão-de-fábrica para administrar tais dados. Desta forma, foi proposto um modelo de banco de dados que pode ser utilizado por sistemas de $B I$ disponibilizados comercialmente por empresas como IBM, Oracle, Microsoft, Seagate, entre outras. No presente artigo foi construído um Data Mart de uma empresa fictícia e utilizado o Analysis Services do Microsoft SQL Server. Por meio dos exemplos dos cubos montados, é possível verificar a facilidade e flexibilidade de tais sistemas de $B I$, conforme prometido na literatura. Sem dúvida, este sistema trará ao gerente um bom suporte para a tomada de direções em busca de melhores resultados para a sua área. Sem informações corretas e no momento certo, não é possível controlar; sem controlar, não é possível gerenciar; e, sem gerenciar, não é possível melhorar (Kueng et al., 2001).

O próximo passo deste trabalho, pretendido pelos autores, é a exploração das funções MDX (multidimensinal expressions) e das ferramentas para Data Mining disponíveis no Analysis Services. Uma outra vantagem do uso do SQL Server 2000 é que seu banco de dados facilitará a integração e incorporação dos dados com os demais sistemas $O L T P$ ou ERP já em funcionamento na empresa, pois apresenta uma solução amplamente aceita nos ambientes empresariais.

\section{Referências Bilbliográficas}

AVISON, D. E.; CUTHBERTSON, C. H.; POWELL, P. The paradox of information systems: strategic value and low status. Journal of Strategic Information Systems, v. 8, n. 4, p. 419-445, 1999.

BÄCK, T. Adaptative business intelligence based on evolution strategies: some application examples of self-adaptative software. Information Sciences, v. 148, n. 1-4, p. 113-121, may. 2002.

BISPO, C. A. F. Uma análise da nova geração de sistemas de apoio à decisão. 1998. 165 p. Dissertação (Mestrado em Engenharia de Produção) - Escola de Engenharia de São Carlos, Universidade de São Paulo, São Carlos, 1998.

BISPO, C. A. F.; CAZARINI, E. W. A nova geração de sistemas de apoio à decisão. In: ENEGEP, 18, 1998, Niterói, Rio de Janeiro, Brasil. Anais... Niterói: ABEPRO, 1998.

CARLSSON, C.; TURBAN, E. DSS: directions for the next decade. Decision Support Systems, v. 33, n. 2, p. 105110, 2002.

COREY, M., ABBEY, M.; ABRAMSON, I.; TAUB, B. Oracle 8i Data Warehouse. 1 ed. Rio de Janeiro: Campus, 2001. $817 \mathrm{p}$.

GRAY, P.; WATSON, H. J. The new DSS: data warehouses, OLAP, MDD and KDD. 1999. Disponível em: $<$ http://hsb.baylor.edu/ramsover/ais.ac.96/papers/graywats.htm>. Acesso em: 20 março 1999.

GRIGORI, D., CASATI, F.; CASTELLANOS, M.; DAYAL, U.; SAYAL, M.; SHAN, M. C. Business Process Intelligence. Computers in Industry, v. 53, n. 3, p. 324-343, 2004.

HARDING, J. A.; YU, B. Information-centred enterprise design supported by a factory data model and data warehousing. Computers in Industry, v. 40, n. 1, p. 23-36, 1999. 
HEINRICHS, J. H.; LIM, J. S. Integrating web-based data mining tolls with business models for knowledge management. Decision Support Systems, v. 35, n. 1, p. 103$112,2003$.

KUENG, P.; WETTSTEIN, T.; LIST, B. A holistic process performance analysis through a performance data warehouse. In: AMERICAS CONFERENCE ON INFORMATION SYSTEMS, 7, 2001, Boston, Massachusetts, USA. Proceedings... Boston, 2001. p. 349-356.

POE, V.; KLAUER, P.; BROBST, S. Building a data warehouse for decision support. 2 ed. Upper Saddle River - NJ: Prentice-Hall PTR, 1998. 285 p.
SHIM, J. P.; WARKENTIN, M.; COURTNEY, J.; POWER, D. J.; SHARDA, R.; CARLSSON, C. Past, present, and future of decision support technology. Decision Support System, v. 33, n. 2, p. 111-126, 2002.

SINGH, H. S. Data Warehouse. 1 ed. São Paulo: Macron Books, 2001. 406 p.

TAN, X.; YEN, D. C.; FANG, X. Web warehousing: web technology meets data warehousing. Technology in Society, v. 25, n. 1, p.131-148, 2003.

ZANCUL, E.; ROZENFELD, H. Sistemas ERP. 1999. Disponível em: <http://www.numa.org.br/conhecimentos/ ERP_V2.html>. Acesso em 16 nov 2000.

\section{A PROPOSAL FOR APPLYING BUSINESS INTELLIGENCE ON THE SHOP FLOOR}

\section{Abstract}

Significant advances have been made on the shop floor in recent decades, involving substantial investments in infrastructure, automation, training and information systems which have transformed the shop floor into a strategic area for companies. Today, the shop floor generates large quantities of data; however, because they are dispersed or disorganized, these data are not used to the best possible advantage as a source of valuable information. In view of this deficiency, this paper proposes the implementation of a Business Intelligence system, using Data Warehouse and OLAP tools specifically applied to the shop floor. Our objective is to develop a system that uses production data, transforming them into information to aid managers in decision-making, thereby enhancing the company's competitiveness. A prototype of the system was built with simulated data to test the viability of this proposal.

Keywords: decision-making support system, business intelligence, data warehouse, OLAP, shop floor. 